\title{
Functional measurement post-stroke via mobile application and body-worn sensor technology
}

\author{
Nancy Fell ${ }^{1}$, Hanna H. True ${ }^{1}$, Brandon Allen ${ }^{2}$, Austin Harris ${ }^{2}$, Jin $\mathrm{Cho}^{2}$, Zhen $\mathrm{Hu}^{2}$, Mina Sartipi ${ }^{2}$, \\ Krystal K. Place ${ }^{1}$, Rebecca Salstrand ${ }^{1}$
}

${ }^{1}$ Department of Physical Therapy, ${ }^{2}$ Department of Computer Science and Engineering, University of Tennessee at Chattanooga, Chattanooga, TN, USA

Contributions: (I) Conception and design: B Allen, J Cho, N Fell, A Harris, Z Hu, M Sartipi, HH True; (II) Administrative support: N Fell, KK Place, R Salstrand, M Sartipi; (III) Provision of study material or patients: B Allen, J Cho, N Fell, A Harris, Z Hu, M Sartipi, HH True; (IV) Collection and assembly of data: N Fell, HH True; (V) Data analysis and interpretation: B Allen, J Cho, N Fell, A Harris, Z Hu, KK Place, R Salstrand, M Sartipi; (VI) Manuscript writing: All authors; (VII) Final approval of manuscript: All authors.

Correspondence to: Dr. Nancy Fell. Physical Therapy Department, University of Tennessee at Chattanooga, 3253, 615 McCallie Avenue, Chattanooga, TN 37403, USA. Email: Nancy-Fell@utc.edu.

Background: Long-term management of individuals post-stroke is essential due to the resultant chronic disability and risk for recurrent stroke. Mobile health technology shows increasing promise to provide cost-effective monitoring and support systems for the patient, caregiver, and healthcare team. Ideally, such systems will include stroke management adherence support, mechanisms to link patients and caregivers to resources, and secure longitudinal data collection with archive that are employed to optimize recovery. However, healthcare providers and computer science application developers must first collaborate to identify meaningful measures and develop methods to reliably gather such data remotely via mobile systems.

Methods: mStroke is a mobile health system composed of two sensors and a mobile application designed to support optimal recovery for stroke survivors. Using the World Health Organization's International Classification of Functioning, Disability and Health model (ICF model), the authors identified 4 measures that are commonly used in the clinic and developed the mobile application features to support remote data collection: National Institutes of Health Stroke Scale (NIHSS) items 5 and 6 (Motor Arm and Leg function), Functional Reach Test (FRT), and 10 Meter Walk Test (10MWT). At a local inpatient rehabilitation facility, each measure was executed with 35 stroke survivors through simultaneous scoring by the mStroke system and standardized clinical assessment. Correlation coefficients were calculated for clinician versus mStroke system scoring.

Results: All four clinical measures significantly correlated with mStroke system app scoring: NIHSS Motor Arm—0.839, $\mathrm{P}<0.001$; NIHSS Motor Leg—0.736, $\mathrm{P}<0.001$; FRT—0.630, $\mathrm{P}<0.01 ; 10 M W T-0.994$, $\mathrm{P}<0.001$.

Conclusions: Results should be approached with caution as significant data skew was present for NIHSS Motor Arm and Motor Leg tests and the FRT results are not strong enough for broad translation. However, positive findings were demonstrated that support further investment in development, refinement, and testing of mobile health systems to provide clinically meaningful remote measurement via mobile technology. The ICF model was a helpful framework for guiding clinician and application developer collaboration and identifying meaningful features for app development.

Keywords: Mobile applications; stroke; stroke rehabilitation; telemedicine

Received: 01 May 2019; Accepted: 15 August 2019; Published: 08 October 2019.

doi: $10.21037 /$ mhealth.2019.08.11

View this article at: http://dx.doi.org/10.21037/mhealth.2019.08.11 


\section{Introduction}

Stroke is the leading cause of serious, long-term disability in adults in the United States. Each year, approximately 795,000 individuals suffer new or recurrent strokes (1). An individual's risk of recurrent stroke increases six-fold after his or her first stroke (2). At an annual cost of more than $\$ 40$ billion (1), the cost of care post-stroke is significant, and earlier hospital discharge is becoming more common (3). The average acute care hospital length of stay post-stroke has decreased dramatically in recent years from 10.2 days in 1989 (3) to 4.7 days in 2014 (1). After discharge from an acute care hospital, patients ideally enter a coordinated, multidisciplinary rehabilitation phase in an inpatient rehabilitation hospital focused on stroke recurrence prevention, restoration of systems impaired by the stroke, and functional return to activities of daily living (4). This early rehabilitation decreases disability and dependency (5). Professional intervention during this stage is restricted to a short time frame, with formal rehabilitation typically ending 3-4 months after the initial stroke (4). Departure from this stage is far from the end of the restorative process, as stroke sequelae have potential for improvement years after the acute management phase ends (6). Whether in an acute care hospital or an inpatient rehabilitation hospital, the brevity of organized, professional care is disproportionate to the long-term effects of stroke.

The objectives of longitudinal post-stroke management are two-fold: to prevent stroke recurrence and provide support toward optimal functional recovery. Early discharge from rehabilitation can leave many functional gains unrealized. Due to the chronicity of stroke disability, these objectives require long-term attention (4,7). Evidence of prolonged disability is apparent in stroke survivors' increased risk of falls (8), sedentary lifestyle (9), decreased use of affected limbs (10), and decreased community integration (4). As stroke prevalence continues to rise, innovative methods must be leveraged to provide patients post-stroke with quality long-term care at a decreased burden of cost.

Mobile health technology shows burgeoning potential to meet this need for cost-effective, long-term care (11-13). With advancements in mobile application and wearable sensor technology, medical and rehabilitation management may be extended beyond the hospital stay. Mobile health may improve both early and standard discharge by allowing continued facilitation of functional recovery in the context of home and community integration (14). A mobile health system for individuals post-stroke must meet a myriad of needs including adherence support, streamlined communication between the patient and healthcare team, early identification and mitigation of complications, and caregiver support. Development of a patient-friendly, clinically useful, and cost-effective mobile health system may provide the long-term support and monitoring needed to reduce stroke recurrence and optimize recovery.

The authors propose a mobile health system called mStroke. This system consists of two wearable sensors and a mobile application (app) designed to be a comprehensive support management system for patients post-stroke. Current market-available, stroke-focused mobile applications have limited utility and focus on providing only one aspect of support, such as exercise plans or clinical measurement tools for healthcare providers $(11,15)$. In the development of this app, healthcare providers collaborated with computer science application developers using the World Health Organization's International Classification of Functioning, Disability, and Health (ICF) model to determine meaningful features as they pertain to individuals who have suffered a stroke. mStroke will be the first stroke-specific, comprehensive system designed to symbiotically benefit the patient, the patient's caregivers, and the healthcare providers. The mobile app will allow personal goal setting and benchmarking; health status monitoring; medication, nutrition, exercise, and activity adherence tracking and support; depression and stroke impact surveys; streamlined communication; and real-time motion monitoring.

This study focuses on the capture of real-time movement data in four common clinical tools used to assess stroke recovery at the body function and activity levels of the ICF model. The National Institutes of Health Stroke Scale (NIHSS) is used to evaluate neurological status (16). Items 5 (Motor Arm) and 6 (Motor Leg) of the NIHSS are used in this study to measure extremity strength at the ICF model body function level. The Functional Reach Test (FRT), a clinical measure at the ICF model activity level, documents how far an individual can forward reach before taking a step (17) and assesses fall risk (18). The 10 Meter Walk Test (10MWT), also an activity level measurement, defines an individual's preferred gait speed $(19,20)$. The purpose of this study was to examine correlations between clinician and mobile app scoring for these tests.

The NIHSS is a tool designed to indirectly measure neurological function with a 15-item impairment scale that covers consciousness, ocular movement, vision, facial muscle function, upper and lower extremity strength, reflexes, coordination, sensory function, neglect, speech, 
and language (21). It was designed to be a practical and efficient tool used at the patient's bedside, and takes approximately 7 minutes to complete (21). The NIHSS is a strong predictor of outcomes after stroke and may influence decisions of future management based on prognosis $(22,23)$. The scale exhibits high concurrent validity to stroke severity (24), high inter- and intrarater reliability in both neurologically-trained and non-neurologically-trained raters (25), and reliability and validity via telemedicine (26). Items 5 (Motor Arm) and 6 (Motor Leg), which test upper and lower extremity strength, were selected for use in this study. Stroke typically affects primarily one side of the body; thus, comparing the strength of both sides may provide valuable information about recovery.

The FRT measures margin of stability, including change in balance performance over time (27). In assessment of patients post-stroke, the FRT has a high intrarater reliability (ICC $=0.89)$ and validity $(\mathrm{r}=0.71)(28)$. The FRT is correlated with the Berg Balance Scale (BBS) ( $r=0.78)$, making it a good stand-in assessment for fall risk when the BBS is not feasible (18). The FRT is well represented in the literature and has documented norms for patients poststroke (29).

The 10MWT is the gait speed assessment of choice for patients "with neurologic conditions who have goals to improve walking speed and have the capacity to change in this area," as substantiated by Moore et al., 2018 (30). The 10MWT has been shown to be a clinically meaningful measure of walking ability with excellent interrater reliability (ICC $=0.998)(31)$, testretest reliability (ICC $=0.94$ ) (19), and good predictive validity of dependence in instrumental activities of daily living $(\mathrm{r}=0.76)(32)$. The $10 \mathrm{MWT}$ has a minimal clinically important difference of $0.16 \mathrm{~m} / \mathrm{s}$ (33). Gait speed has also been correlated with the BBS $(r=0.627)(31)$, which indicates fall risk in patients post-stroke (34). Gait speed is also a valuable method of gauging community access. For example, the average speed required to cross the street during a signal change is $0.49 \mathrm{~m} / \mathrm{s}(35)$. Based on this and other known gait speeds required for safe ambulation in the community, functional ambulation classes have been defined. Gait speeds between $0.4-0.8 \mathrm{~m} / \mathrm{s}$ allow limited community ambulation, while speeds greater than $0.8 \mathrm{~m} / \mathrm{s}$ allow regular community ambulation $(36,37)$. Transition to a higher functional ambulation class is indicative of improvement in function and quality of life (20).

\section{Methods}

\section{Materials}

$\mathrm{mStroke}$ is a wireless system composed of a mobile app installed on an iPad and two NODE sensors worn on the chest and/or arm or leg. Each sensor is a $25.4 \mathrm{~mm}$ diameter cylinder with a length of $83.8 \mathrm{~mm}$. It is a low-power, lowlatency device with modular platform that uses Bluetooth low-energy protocol to communicate with a computer/ smart phone base station. The sensor platform is an inertial motion unit (IMU) containing a 3 -axis accelerometer, magnetometer, and gyroscope. It sends motion data to the base station app at up to 120 samples per second with a range of up to $50 \mathrm{~m}$.

\section{Participants}

This study was approved by hospital and university institutional review boards (IRB approval \#14-023). After performing preliminary testing on healthy individuals, 35 patients post-stroke (21 males, 14 females) at a local inpatient rehabilitation hospital were recruited for participation. Informed consent from all participants and their physicians was obtained prior to study enrollment. Nineteen participants presented with left hemiparesis, 11 with right hemiparesis, and 5 with mild bilateral hemiparesis. To be considered for inclusion, participants were required to have suffered a stroke within the last 3 months, comprehend and follow 3 -step commands, and ambulate 10 meters without rest and with no more than minimal physical assistance with or without an assistive device. Patients were excluded from the study if they had previous neurological diagnosis(es), neglect syndrome, aphasia, apraxia, amputation, or surgery within the last 6 months. Upon vital sign screening, patients were excluded if their resting heart rate was greater than 90 beats per minute and/or blood pressure was greater than $180 / 90 \mathrm{mmHg}$. Patients were also excluded if they displayed a blood pressure drop of more than $20 \mathrm{mmHg}$ upon standing with symptoms of blurred vision, cognitive decline, and/or dizziness.

\section{Procedures}

Heart rate and blood pressure were assessed before data collection. Upon inclusion based on the criteria above, participants provided demographic information and were fitted with the sensors. Participants were assigned a subject 
Table 1 NIHSS motor arm and motor leg trials clinician and mobile app score distributions

\begin{tabular}{lcc}
\hline NIHSS score & Motor Arm & Motor Leg \\
\hline 0 & 157 & 145 \\
1 & 20 & 43 \\
2 & 5 & 10 \\
3 & 10 & 0 \\
4 & 6 & 0 \\
\hline
\end{tabular}

identification number, which they were instructed to enter into the mStroke app; iPad and sensor donning/doffing assistance was provided as necessary.

The NIHSS Motor Arm and Motor Leg tests were administered per the protocol provided by the National Institutes of Health (38). In the NIHSS Motor Arm test, one sensor was placed on the distal humerus. In a seated position, the participant's arm was placed at 90 degrees of shoulder flexion. The participant was instructed to hold their arm in this position for 10 seconds. If the limb exhibited no effort against gravity in the original testing position, the arm was positioned in an anatomic neutral position at their side and the patient was asked to try to lift the limb from this position. Three repetitions were performed on each upper extremity beginning with the less affected side. In the NIHSS Motor Leg test, one sensor was placed on the ankle just proximal to the malleolus. The patient assumed a supine position. To protect the low back, the contralateral lower extremity was placed in hip and knee flexion with the foot planted on the bed. The participant's tested leg was placed at approximately 30 degrees of hip flexion with full knee extension. The participant was instructed to hold their leg in this position for 5 seconds. If the limb exhibited no effort against gravity in the original testing position, the patient was asked to try to lift the limb from a supine resting position. Three repetitions were performed on each lower extremity beginning with the less affected side.

In the FRT, two sensors were used, one on the wrist and one on the sternum. Per the testing protocol described by Duncan et al. 1990, participants began in standing and were instructed to lift their less affected arm to 90 degrees of shoulder flexion and reach as far forward as possible without moving their feet or losing their balance (27). Three repetitions were performed.

In the 10MWT, two sensors were used, one on each ankle just proximal to the malleoli. Per the testing protocol described by Steffen \& Seney 2008, from a standing position, participants were asked to walk at a self-selected comfortable pace over a level surface in a controlled environment (39). Participants were provided assistance from a licensed physical therapist following standard precautions including use of a gait belt and physical guarding.

All tests were clinically scored by the physical therapist using the standard test protocols. Simultaneously, IMU data were collected by the sensor(s) and transferred to the mobile app, which scored the performance. Clinician and app scores for each trial were documented.

\section{Analysis}

Data were transferred from individual participant data collection forms to an Excel spreadsheet, then imported into SPSS version 23 (40). Nonparametric (small number of participants; NIHSS ordinal data) Spearman Rho correlation coefficients were calculated between clinician and mobile app scores for each activity.

\section{Results}

The mobile app failed in data collection for two participants, thus their data were discarded, and results analyzed for 33 participants. For the FRT and 10MWT, three trials were performed with each participant: 99 total trials for each FRT and 10MWT. For the NIHSS Motor Arm and Leg tests, three trials were performed on the lesser affected and more affected limbs with each participant: 198 total trials for each Motor Arm test and Motor Leg test. For each of the NIHSS Motor Arm and Leg tests on the participant's lesser affected limbs, the resulting NIHSS score was 0 (normal function) for all trials. Thus, the distribution of NIHSS scores (Likert scale range 0-4) were strongly skewed to the 0 score for both Motor Arm and Motor Leg trials. Table 1 shows the NIHSS score distributions.

Clinician and mobile app scores Spearman Rho correlation coefficient results are listed in Table 2.

\section{Discussion}

Significant correlation between clinician and mobile app scores for all four tests supports the authors' objectives to create a mobile health app that provides clinicians with objective movement data to inform and track recovery. These findings have valuable safety implications in that remotely gathered information could be used to 
Table 2 Correlation between clinician and mobile app scores

\begin{tabular}{lc}
\hline Clinical measure & Spearman Rho \\
\hline NIHSS Motor Arm & $0.839^{\star}$ \\
NIHSS Motor Leg & $0.736^{\star}$ \\
FRT & $0.630^{\star *}$ \\
1OMWT & $0.994^{*}$ \\
\hline
\end{tabular}

*, significant at $\mathrm{P}<0.001$; ${ }^{\star}$, significant at $\mathrm{P}<0.01$. FRT, Functional

Reach Test; 10MWT, 10 Meter Walk Test.

identify potentially harmful changes in patient status and trigger timely healthcare interventions, thus mitigating complications. These results support investment in additional development, refinement, and testing of mobile health systems to provide clinically meaningful remote measurement via mobile technology.

The best results were demonstrated for the 10MWT (0.994; $\mathrm{P}<0.001$ ), which is consistent with prior research documenting the reliability of gait speed data collected via body worn sensors (41-43). For the NIHSS Motor Arm and Motor Leg tests, the results are promising but far from conclusive ( 0.839 and $0.736 ; \mathrm{P}<0.001$, respectively). A chief limitation in the NIHSS-related analyses were the aforementioned data skew toward NIHSS Motor Arm and Motor Leg score of 0. Additionally, participant inclusion criteria included the ability to ambulate 10 meters without rest and minimal physical assistance. This resulted in excluding participants functioning at lower mobility levels, many of whom are likely to have more moderate to severe motor impairment of the affected upper and lower extremities. Additionally, the NIHSS Motor Arm and Leg tests mobile apps employed a rule-based algorithm for scoring. The authors hypothesize that a machinebased approach, while labor intensive in development, may significantly improve correlations.

The FRT results were disappointing and represent the challenge of designing a mobile app that uses data from two sensors to capture a discrete measurement (forward functional reach) obtained during a complex and highly variable functional activity. The forward functional reach involves multi-planar movement at the ankles, knees, hips, trunk, and shoulder complex. Thus, while the correlation results for the FRT were statistically significant $(0.630$; $\mathrm{P}<0.01$ ), they are insufficient for clinical use. The primary goal of the FRT remote measurement app was to reliably capture a clinically relevant measure that has predictive capacity. Documenting an individual's fall risk is important post-stroke. The authors propose that the Timed Up and Go (TUG) test may be a better clinical measurement to translate to mobile systems for remote data collection as it includes both fast self-selected gait speed and is predictive of fall risk (44). The TUG test measures the time required for an individual to stand up from a standard-height chair, walk 3 meters, turn 180 degrees, walk to the chair, turn 180 degrees, and sit down (45). Clinically, the TUG has an excellent test-retest reliability (ICC $=0.96)(19)$.

Future research should repeat this study with a larger number of participants who present with greater diversity of sensory and/or motor impairments as well as a range of functional abilities. Future studies should also explore caregiver collaboration with focus on quantifying and refining the user-friendliness of these types of apps, especially as it relates to usage in elderly and disabled populations. Next steps for mStroke specifically will include identification and further development of additional key measures and features to be included in the app, such as adherence support, streamlined communication between the patient and healthcare team, methods for early identification and mitigation of complications, and caregiver support. The NIHSS Motor Arm and Motor Leg tests will receive further attention; namely, the employment of machine-learning in app development.

In conclusion, mobile applications such as mStroke are necessary to provide the long-term support and monitoring needed to reduce stroke recurrence and optimize recovery. This study represents the development of a few of many features within the mStroke system. The results support additional investment in mobile health technology as a viable and affordable way to improve the quality and efficiency of post-stroke care through remote monitoring, ongoing treatment and assessment, and communication links between patients and their healthcare providers. Additionally, information gathered through mobile health applications may contribute to longitudinal analyses regarding recovery and stroke recurrence after initial stroke.

\section{Acknowledgments}

Funding: This work supported by National Institutes of Health through grant 1R15EB015700-01A1.

\section{Footnote}

Conflicts of Interest: The authors have no conflicts of interest to declare. 
Ethical Statement: The authors are accountable for all aspects of the work in ensuring that questions related to the accuracy or integrity of any part of the work are appropriately investigated and resolved. This study was approved by the University of Tennessee at Chattanooga Institutional Review Board (\#14-023). The outcomes of this study did not directly influence the future management of the participants.

\section{References}

1. Benjamin EJ, Virani SS, Callaway CW, et al. Heart Disease and Stroke Statistics-2018 Update: A Report From the American Heart Association. Circulation 2018;137:e67-e492.

2. Hardie K, Hankey GJ, Jamrozik K, et al. Ten-year risk of first recurrent stroke and disability after first-ever stroke in the Perth Community Stroke Study. Stroke 2004;35:731-5.

3. Hall MJ, Levant $S$, DeFrances CJ. Hospitalization for stroke in U.S. hospitals, 1989-2009. NCHS Data Brief 2012;(95):1-8.

4. Winstein CJ, Stein J, Arena R, et al. Guidelines for Adult Stroke Rehabilitation and Recovery: A Guideline for Healthcare Professionals From the American Heart Association/American Stroke Association. Stroke 2016;47:e98-e169.

5. Stroke Unit Trialists' Collaboration. Organised inpatient (stroke unit) care for stroke. Cochrane Database Syst Rev 2013;(9):CD000197.

6. Ferrarello F, Baccini M, Rinaldi LA, et al. Efficacy of physiotherapy interventions late after stroke: a metaanalysis. J Neurol Neurosurg Psychiatry 2011;82:136-43.

7. Winstein CJ, Merians AS, Sullivan KJ. Motor learning after unilateral brain damage. Neuropsychologia 1999;37:975-87.

8. Batchelor FA, Mackintosh SF, Said CM, et al. Falls after stroke. Int J Stroke 2012;7:482-90.

9. English C, Healy GN, Coates A, et al. Sitting and Activity Time in People With Stroke. Phys Ther 2016;96:193-201.

10. Nakayama H, Jorgensen HS, Raaschou HO, et al. Compensation in recovery of upper extremity function after stroke: the Copenhagen Stroke Study. Arch Phys Med Rehabil 1994;75:852-7.

11. Burridge JH, Lee ACW, Turk R, et al. Telehealth, Wearable Sensors, and the Internet: Will They Improve Stroke Outcomes Through Increased Intensity of Therapy, Motivation, and Adherence to Rehabilitation Programs? J
Neurol Phys Ther 2017;41 Suppl 3:S32-S38.

12. Dobkin BH, Dorsch A. The promise of mHealth: daily activity monitoring and outcome assessments by wearable sensors. Neurorehabil Neural Repair 2011;25:788-98.

13. Pugliese M, Ramsay T, Johnson D, et al. Mobile tabletbased therapies following stroke: A systematic scoping review of administrative methods and patient experiences. PLoS One 2018;13:e0191566.

14. Langhorne P, Baylan S. Early supported discharge services for people with acute stroke. Cochrane Database Syst Rev 2017;7:CD000443.

15. Halket D, Singer J, Balucani C, et al. Mobile Applications for Stroke Prevention: a Survey of Physicians' Perspectives. J Mob Technol Med 2017;6:7-13.

16. Josephson SA, Hills NK, Johnston SC. NIH Stroke Scale reliability in ratings from a large sample of clinicians. Cerebrovasc Dis 2006;22:389-95.

17. Tyson SF, DeSouza LH. Reliability and validity of functional balance tests post stroke. Clin Rehabil 2004;18:916-23.

18. Smith PS, Hembree JA, Thompson ME. Berg Balance Scale and Functional Reach: determining the best clinical tool for individuals post acute stroke. Clin Rehabil 2004;18:811-8.

19. Flansbjer UB, Holmback AM, Downham D, et al. Reliability of gait performance tests in men and women with hemiparesis after stroke. J Rehabil Med 2005;37:75-82.

20. Schmid A, Duncan PW, Studenski S, et al. Improvements in speed-based gait classifications are meaningful. Stroke 2007;38:2096-100.

21. Brott T, Adams HP Jr, Olinger CP, et al. Measurements of acute cerebral infarction: a clinical examination scale. Stroke 1989;20:864-70.

22. Adams HP Jr, Davis PH, Leira EC, et al. Baseline NIH Stroke Scale score strongly predicts outcome after stroke: A report of the Trial of Org 10172 in Acute Stroke Treatment (TOAST). Neurology 1999;53:126-31.

23. Muir KW, Weir CJ, Murray GD, et al. Comparison of neurological scales and scoring systems for acute stroke prognosis. Stroke 1996;27:1817-20.

24. Brott T, Marler JR, Olinger CP, et al. Measurements of acute cerebral infarction: lesion size by computed tomography. Stroke 1989;20:871-5.

25. Goldstein LB, Samsa GP. Reliability of the National Institutes of Health Stroke Scale. Extension to nonneurologists in the context of a clinical trial. Stroke 1997;28:307-10.

26. Meyer BC, Raman R, Chacon MR, et al. Reliability of site- 
independent telemedicine when assessed by telemedicinenaive stroke practitioners. J Stroke Cerebrovasc Dis 2008; 17:181-6.

27. Duncan PW, Weiner DK, Chandler J, et al. Functional reach: a new clinical measure of balance. J Gerontol 1990;45:M192-7.

28. Duncan P, Studenski S, Richards L, et al. Randomized clinical trial of therapeutic exercise in subacute stroke. Stroke 2003;34:2173-80.

29. Outermans JC, van Peppen RP, Wittink H, et al. Effects of a high-intensity task-oriented training on gait performance early after stroke: a pilot study. Clin Rehabil 2010;24:979-87.

30. Moore JL, Potter K, Blankshain K, et al. A Core Set of Outcome Measures for Adults With Neurologic Conditions Undergoing Rehabilitation: a Clinical Practice Guideline. J Neurol Phys Ther 2018;42:174-220.

31. Wolf SL, Catlin PA, Gage K, et al. Establishing the reliability and validity of measurements of walking time using the Emory Functional Ambulation Profile. Phys Ther 1999;79:1122-33.

32. Tyson S, Connell L. The Psychometric properties and clinical utility of measures of walking and mobility in neurological conditions: a systematic review. Clin Rehabil 2009;23:1018-33.

33. Tilson JK, Sullivan KJ, Cen SY, et al. Meaningful gait speed improvement during the first 60 days poststroke: minimal clinically important difference. Phys Ther 2010;90:196-208.

34. Doğan A, Mengulluoglu M, Ozgirgin N. Evaluation of the effect of ankle-foot orthosis use on balance and mobility in hemiparetic stroke patients. Disabil Rehabil 2011;33:1433-9.

35. Andrews AW, Chinworth SA, Bourassa M, et al. Update on distance and velocity requirements for community

doi: $10.21037 /$ mhealth.2019.08.11

Cite this article as: Fell N, True HH, Allen B, Harris A, Cho J, Hu Z, Sartipi M, Place KK, Salstrand R. Functional measurement post-stroke via mobile application and body-worn sensor technology. mHealth 2019;5:47. ambulation. J Geriatr Phys Ther 2010;33:128-34.

36. Perry J, Garrett M, Gronley JK, et al. Classification of walking handicap in the stroke population. Stroke 1995;26:982-9.

37. Bowden MG, Balasubramanian CK, Behrman AL, et al. Validation of a speed-based classification system using quantitative measures of walking performance poststroke. Neurorehabil Neural Repair 2008;22:672-5.

38. NIH Stroke Scale. National Institutes of Health. 2003. Available online: https:/www.stroke.nih.gov/documents/ NIH_Stroke_Scale_508C.pdf, accessed December 4, 2018.

39. Steffen T, Seney M. Test-retest reliability and minimal detectable change on balance and ambulation tests, the 36item short-form health survey, and the unified Parkinson disease rating scale in people with parkinsonism. Phys Ther 2008;88:733-46.

40. IBM Corp. Released 2015. IBM SPSS Statistics for Windows, Version 23.0. Armonk, NY: IBM Corp.

41. Qiu S, Wang Z, Zhao H, et al. Using Body-Worn Sensors for Preliminary Rehabilitation Assessment in Stroke Victims with Gait Impairment. IEEE Access 2018;6:31249-58.

42. Saremi K, Marehbian J, Yan X, et al. Reliability and validity of bilateral thigh and foot accelerometry measures of walking in healthy and hemiparetic subjects. Neurorehabil Neural Repair 2006;20:297-305.

43. Fulk GD, Sazonov E. Using sensors to measure activity in people with stroke. Top Stroke Rehabil 2011;18:746-57.

44. Persson CU, Danielsson A, Sunnerhagen KS, et al. Timed Up \& Go as a measure for longitudinal change in mobility after stroke - Postural Stroke Study in Gothenburg (POSTGOT). J Neuroeng Rehabil 2014;11:83.

45. Podsiadlo D, Richardson S. The timed "Up \& Go": a test of basic functional mobility for frail elderly persons. J Am Geriatr Soc 1991;39:142-8. 